\title{
TESTS OF AN ACTIVE, BROAD-BAND ANTENNA ARRAY
}

\author{
I. N. Bubnov*, A. A. Konovalenko*, I. S. Falkovich*, H. O. Rucker ${ }^{\dagger}$, \\ A. A. Gridin*, N. N. Kalinichenko*, A. P. Reznik*, S. V. Stepkin*, \\ D. V. Mukha*, V. V. Dorovskyy*, and A. Lecacheux ${ }^{\ddagger}$
}

\begin{abstract}
In this paper, test results from a 25-element active antenna prototype array operating in the frequency range of $10-70 \mathrm{MHz}$ are presented. Observations of radio emission from different sources: solar sporadic radio emission and powerful cosmic radio sources including their ionosphere scintillation, demonstrate the high effectiveness of the system due to the Galactic background limited sensitivity and high dynamic range of the antenna amplifier (noise immunity). This demonstrates the capability of this 25-element active antenna array to engage in a wide range of unique wide band radio astronomical observations of solar system objects that do not require high sensitivity and angular resolution.
\end{abstract}

\section{Introduction}

In recent years interest in low frequency radio astronomy (meter and decameter wavelength bands) has steadily increased worldwide. According to the special program of the National Academy of Sciences of Ukraine (NASU) the Institute of Radio Astronomy (IRA NASU) has started a stepwise development of the Giant Ukrainian Radio Telescope (GURT) - the Ukrainian Radio Telescope of a new age. Since 2000, the GURT project has been developed by the IRA NASU. The first aim of the GURT project is to build a lowfrequency wideband (10 to $60 \mathrm{MHz}$ ) antenna array. The GURT project is the continuation of the UTR-2 project [Braude et al., 1978]. The UTR-2 project was culminated in 1970 with the creation of the large T-shape decametric radio telescope with an effective area of about 150, 000 square meters. At the end of 2000, in the first stage of the GURT project, a thirty-element antenna array was elaborated and built. The effective wide-band (10 to

\footnotetext{
* Institute of Radio Astronomy, Ukrainian Academy of Sciences, Chervonopraporna 4, 61002, Kharkiv, Ukraine

† Space Research Institute, Austrian Academy of Sciences, Schmiedlstrasse 6, A-8042 Graz, Austria

‡LESIA, Observatoire de Paris, UMR CNRS 8109, 92195 Meudon, France
} 
$60 \mathrm{MHz}$ ) active antenna element has been developed. Thin horizontal active dipoles with arm lengths of $1.5 \mathrm{~m}$ and a height of $3.5 \mathrm{~m}$ were used as antenna elements. As the thirtyelement array had the same configuration as that of the UTR-2 section, it was possible to compare their characteristics. The tests carried out in 2001 showed the high sensitivity and interference immunity of the thirty-element active array [Konovalenko et al., 2005]. In the second stage of the GURT project, which is described in this paper, the dipole and preamplifier designs, and the match between the dipole and preamplifier have been improved to obtain the maximum possible ratio between the antenna temperature due to Galactic noise $T_{p r e}$ and the noise temperature of the preamplifier $T_{s k y}^{a}$. We set ourself the task to obtain $\alpha=10 \log _{10}\left(T_{s k y}^{a} / T_{\text {pre }}\right) \approx 10 \mathrm{~dB}$ over the whole 10 to $70 \mathrm{MHz}$ range. We did not set ourself the task to achieve similar results at higher frequencies because the strong signals of FM radio stations between 68 and $75 \mathrm{MHz}$ make high-quality radio astronomical observations impossible. Considerable attention has been given to the total efficiency of the dipole at frequencies from 10 to $30 \mathrm{MHz}$. In this frequency range it is possible to study important astrophysical problems and there is synergy with the UTR-2 and URAN antennas.

\section{The Active Dipole for the Low-frequency Array}

An antenna element for large low-frequency arrays must satisfy several requirements. Firstly, it must be mechanically simple, inexpensive, reliable, and be able to endure extreme weather conditions like strong winds, blizzards, cold, heat, and humidity. Secondly, the antenna element is required to have wide pattern in the $\mathrm{E}$ and $\mathrm{H}$ planes to provide wide field of view of the radio telescope. We managed to design a dipole that conforms to all requirements mentioned above. The dipole is $1.4 \mathrm{~m} \mathrm{long}$, the width near the feed point is $0.9 \mathrm{~m}$, and the height above ground is $1.6 \mathrm{~m}$. The dipole is constructed from $5 \mathrm{~mm}$ coaxial cable (the only part of the cable which is used in the screen) placed into larger diameter plastic pipe to make the structure more rigid.Calculated antenna pattern of the dipole is shown in Figure 1

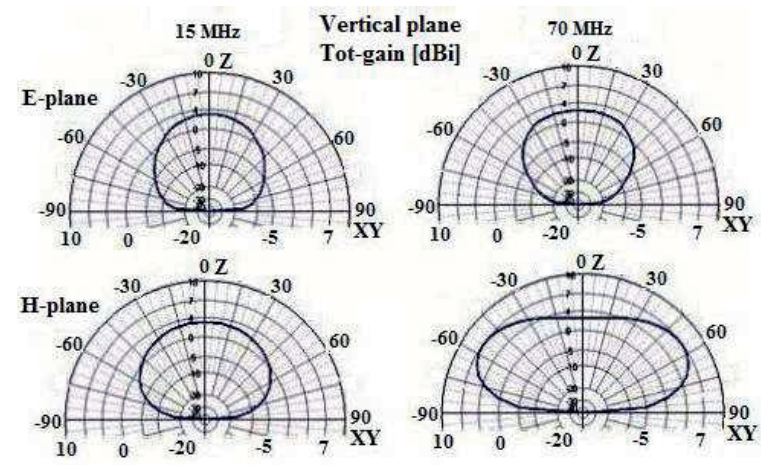

Figure 1: The dipole pattern in $E$ and $H$ planes calculated for $h=1.6 \mathrm{~m}$. 
An antenna amplifier for an active dipole must have low noise, high linearity and high enough gain. The fulfillment of the last condition means that the contribution of the subsequent devices to the noise temperature of the radio telescope can be ignored. Also, at low frequencies the presence of very strong radio-frequency interference (RFI) makes linearity an extremely important characteristic. Linearity can be quantified in terms of the input second-order intercept point IIP2 and the input third-order intercept point IIP3. If the values of IIP2 and IIP3 are not large enough, the sensitivity of observations is limited by the level of the wideband non-stationary intermodulation products for an arbitrary large time of integration. The amplifier is made on the basis of a two-stage push-pull scheme. Each stage is a scheme with common base and low-noise transformer feedback. In the first stage a low-noise bipolar transistor BFR96 with the current of the collector $I_{c}=10 \mathrm{~mA}$ is used. In the second stage a transistor BFR96 with $I_{c}=40 \mathrm{~mA}$ is used to provide high dynamic range in combinations of the third order (IIP3). The pushpull amplifier construction provides the dynamic range on the second order combinations (IIP2). The amplifier gain is $18 \mathrm{~dB}$, the output VSWR of the preamplifier is less than 1.5 in the operation frequency range.

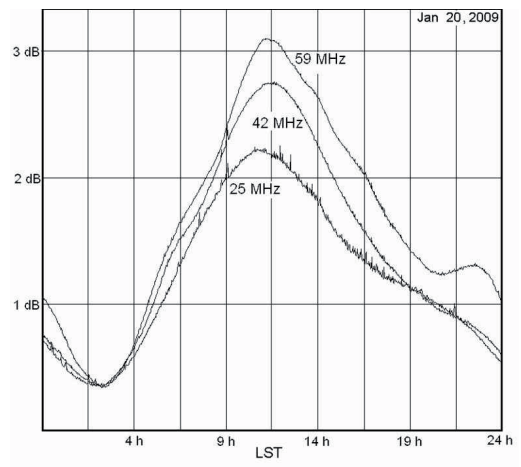

Figure 2: The diurnal variations of the antenna temperature due to Galactic noise at 25, 42 and $59 \mathrm{MHz}$ which were obtained by a digital spectral processor. LST (Local Stellar Time).

Figure 2 shows diurnal variations of $T_{s k y}^{a}$ at 25, 42 and $59 \mathrm{MHz}$, which we obtained by using DSP (digital spectral processor) developed in the IRA NASU [Kozhyn et al., 2007]. The data represent the ability of the dipole to provide Galactic noise-limited operation in the frequency range 10 to $70 \mathrm{MHz}$. It is known that the $\gamma$ is the ratio of maximum to minimum temperature $T_{s k y}^{a}$ and takes a maximum value for an "ideal" active dipole that has a noiseless amplifier and is placed over a perfectly conducting ground. Krymkin [1971] carried out experiments at the UTR-2 observatory to estimate the ratio $\gamma$ for the "real" half-wave dipole which had a low noise amplifier and was placed over a large ground screen. Krymkin found that $\gamma_{\max }$ (maximum meaning of $\gamma$ ) ranges from 2.4 to $3.0 \mathrm{~dB}$ over the frequency range of interest. The ratio $\gamma$ is known to depend on latitude and frequency. Also, $\gamma$ tends to unity $(0 \mathrm{~dB})$ as mismatch or the preamplifier noise increases. In the experiment the ratio $\gamma$ was equal to $2.7 \mathrm{~dB}$ at $59 \mathrm{MHz}$, which is to say that our dipole is 
close to ideal at this frequency. At the lower frequencies (42 $\mathrm{MHz}$ and $25 \mathrm{MHz}$ ), the ratio $\gamma$ also remains high enough $(2.4 \mathrm{~dB}$ and $1.9 \mathrm{~dB}$, correspondingly). Furthermore, as the maximum of $T_{s k y}^{a}$ was obtained in the daytime, when the absorption in the ionosphere is rather strong, it can be shown by a simple calculation [Krymkin, 1971] that these values were underestimated by $0.15 \mathrm{~dB}$ and $0.3 \mathrm{~dB}$ at $42 \mathrm{MHz}$ and $25 \mathrm{MHz}$, respectively.

Figure 3 displays the sample dynamic spectrum of the type III solar radio-burst as obtained by a digital spectral processor at the dipole output. It is seen that the active dipole operates effectively over the whole 10 to $70 \mathrm{MHz}$ range, solar radio emission revealing itself in the time/frequency plane as dark regions which are prominent above the noise level (white color).

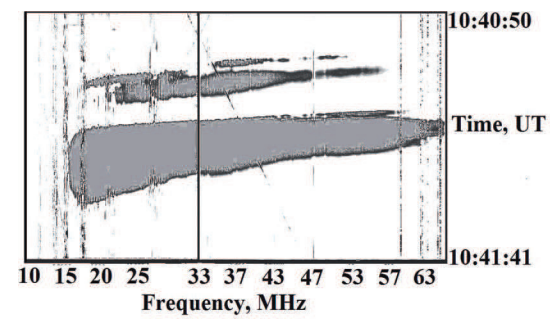

Figure 3: Time - frequency representation of a type III solar radio-burst (i.e. the so-called 'dynamic spectrum') which was obtained by a digital spectral processor at the dipole output.

\section{Antenna Array}

The antenna array represents a square construction and consists of five rows along the East-West line with five active dipoles in each row. The distance between active dipole centers along and across rows equals $3.75 \mathrm{~m}$. All the active dipoles in the antenna array are elevated by $1.6 \mathrm{~m}$ above the ground and oriented under the angle 45 degrees to the EastWest direction. The functional scheme of single polarization 25-elements active antenna array is presented in Figure 4.

The signals from the broadband high-linear active dipoles which form the array come to the analog beamformer via the coaxial cable PK-75-4-11 of equal lengths (L1=24 m). Each row is phased by the analog beamformer that provides 17 beam positions. Rows are combined by general analog beamformer. All six analog beamformers are made by the same scheme. To implement beamforming delays, we used various combinations of PK-75-213 coaxial cable. The commutation element of the analog beamformer is the high-frequency relay G6Y. The power combiners of the analog beamformer are made like a Transmission-Line Transformers (TLTs) [Sevick, 2004]. The signal comes to the input of the amplifier with the gain factor of $18 \mathrm{~dB}$. Then the signal goes from the output of the amplifier to the laboratory building via the backbone cable PK-75-7. The laboratory building is situated at a distance of $200 \mathrm{~m}$ from the antenna array. The directional filter [Abranin et al., 1990], the analog receiver and DSP [Kozhyn et al., 2007] are placed 


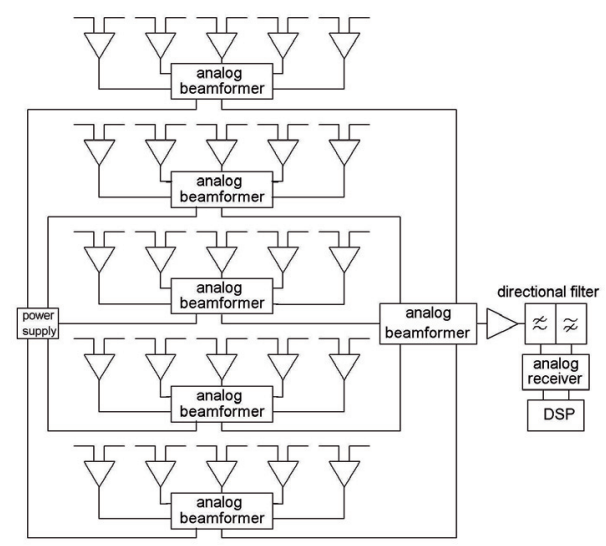

Figure 4: The functional scheme of single polarization 25-elements active antenna array.

there. The directional filter divides the working band into two sub-bands (channels) with crossover frequency of $33 \mathrm{MHz}$. The channel number 1 is below $32 \mathrm{MHz}$ and the channel number 2 is above $34 \mathrm{MHz}$. We applied TLTs (transformers) in this directional filter. The signals go from the outputs of the directional filter to the inputs of the analog receiver where they are additionally amplified. The analog receiver of the 1-st channel contains the anti-aliasing low-pass filter with the cutoff frequency of $32 \mathrm{MHz}$. The second channel of the analog receiver contains the high-pass filter with the cutoff frequency of $35 \mathrm{MHz}$ and the anti-aliasing low-pass filter with the cutoff frequency of $64 \mathrm{MHz}$. The choice of filters is determined by the operational band of DSP [Kozhyn et al., 2007]. This DSP works in the band from 0 up to $33 \mathrm{MHz}$ with the sampling frequency of $66 \mathrm{MHz}$. Each channel of the analog receiver has individual gain from 6 up to $36 \mathrm{~dB}$. The gain factor is changed by the discrete attenuator with a step of $2 \mathrm{~dB}$. The measurements of the brightness temperature of the cosmic radio emission, solar sporadic radio emission, and powerful cosmic radio sources were conducted by antenna array described above. A total power measurements conducted in a 24 hours period with declination of $59^{\circ}$ are shown in Figure 5. Our antenna array has sufficient sensitivity and directional selectivity to mark out the source Cassiopeia A from the total power measurements on the frequency of $60 \mathrm{MHz}$. The sensitivity of the 25-element antenna array is not enough to mark out the source Cassiopeia A from total power measurements on the frequencies below $50 \mathrm{MHz}$ because intensity of the galactic background is grown and the antenna pattern is widened. But we can see the ionospheric scintillations of Cassiopeia-A (Figure 5, left) even at the frequency $30 \mathrm{MHz}$ when the radio source is in the antenna pattern.

The principal antenna array parameters are the following:

- frequency range is $10 \mathrm{MHz}$ to $70 \mathrm{MHz}$;

- sector of electronic tracking is $70^{\circ}$ from zenith for both coordinates; 


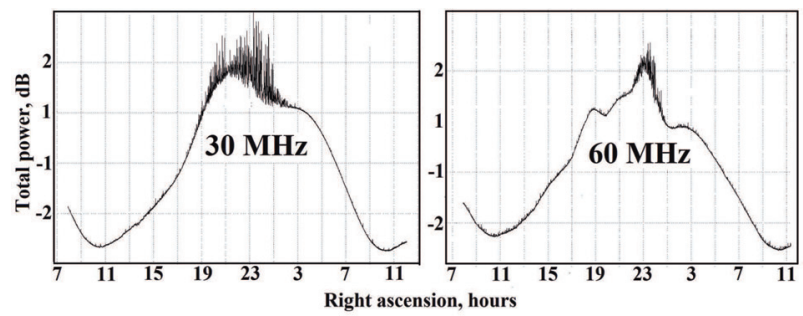

Figure 5: The total power for the active antenna array section with 25-element at $30 \mathrm{MHz}$ and $60 \mathrm{MHz}$. The antenna array section pointing North at a zenith angle of $9^{\circ}$.

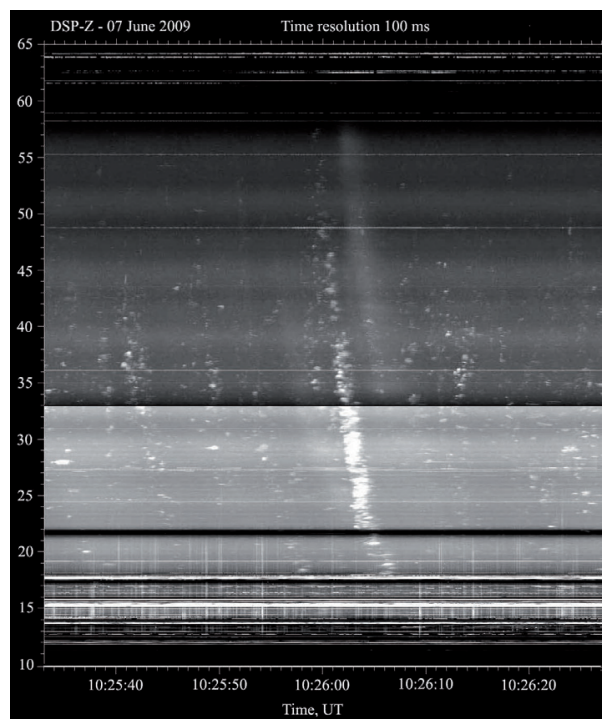

Figure 6: The dynamic spectrum of the type IIIb-III burst 7 June 2009 seen in the range of 10-65 MHz.

- total antenna array size is $18.75 \mathrm{~m} \times 18.75 \mathrm{~m}$;

- antenna array pattern has a width of $20^{\circ} \times 20^{\circ}$ at the frequency $40 \mathrm{MHz}$.

\section{Conclusion}

The 25-element active antenna array described above, which is a part of the GURT radio Telescope project, has limited effective area and angular resolution. Nevertheless, it is sufficient for conducting wide-band radio astronomical observations of solar system objects that do not require high sensitivity and angular resolution. As an example of 
using the developed active, antenna array, the dynamic spectra of a solar Type IIIbIII burst, which was synchronously measured by a two-channel DSP (the channels are superimposed), is shown in Figure 4. The results obtained so far demonstrate that the 25-element active antenna array is quite capable of performing important observations of solar system radiation in the range of $10-70 \mathrm{MHz}$.

\section{References}

Abranin, E. P., Yu. M. Bruck, and A. A. Konovalenko, Highly linear broadband amplifiers with lossless feedback Part 2, Exp. dev. Int. J. Electr., 69, 345-357, 1990.

Braude, S. Ya., A. V. Megn, B. P. Ryabov, N. K. Sharykin, and J. N. Zhouck, Decametric survey of discrete sources in the northern sky: I. The UTR-2 radio telescope. Experimental techniques and data processing, Astrophys. Space Sci., 54, 3-36, 1978.

Konovalenko, A. A., I. S. Falkovich, N. N. Kalinichenko, A. A. Gridin, I. N. Bubnov, A. Lecacheux, C. Rosolen and H. O. Rucker, Thirty-Element Active Antenna Array as a Prototype of a Huge Low-Frequency Radio Telescope, Exp. Astron., 16, 3, 149-164, 2003.

Kozhyn, R.V., V.V. Vynogradov, and D. M. Vavriv, Low-noise, high dynamic range digital receiver/spectrometer for radio astronomy applications, Proceedings The Sixth International Kharkov Symposium on Physics and Engineering of Microwaves, Millimeter and Submillimeter Waves and Workshop on Terahertz Technologies, Kharkov, Ukraine, 2, 736-738, 2007.

Krymkin, V.V., The Spectrum of Background Low-Frequency Radio Emission, Radiophys. Quant. Electr., 14, 161-164, 1971.

Sevick, J. A., Simplified Analysis of the Broadband Transmission Line Transformer, High Freq. Electr., 48-53, 2004. 
